\title{
RESULTADOS CLIINICOS E RADIOGRÁFICOS DO TRATAMENTO CIRÚRGICO DA ESCOLIOSE PARALITICA NA MIELOMENINGOCELE
}

\author{
CLINICAL AND RADIOGRAPHIC RESULTS OF SURGICAL TREATMENT \\ OF PARALYTIC SCOLIOSIS IN MYELOMENINGOCELE
}

\section{RESULTADOS CLIINICOS Y RADIOGRÁFICOS DEL TRATAMIENTO QUIRÚRGICO DE LA ESCOLIOSIS PARALITICA EN LA MIELOMENINGOCELE}

Marcos Gassen Martins ${ }^{1}$, Luís Eduardo Munhoz da Rocha ${ }^{2}$, Carlos Abreu de Aguiar ${ }^{3}$, Edson Pudles ${ }^{4}$, Nadio Sanzovo ${ }^{5}$

\begin{abstract}
RESUMO
Objetivo: Avaliar os resultados clínicos e radiográficos do tratamento cirúrgico da escoliose paralítica na mielomeningocele. Métodos: Estudo retrospectivo mediante revisão de prontuários e radiografias dos pacientes portadores de mielomeningocele, tratados cirurgicamente entre os anos de 1999 e 2009. Resultados: Foram analisados os prontuários e radiografias de 29 pacientes. A média de idade no momento da cirurgia foi de 12,2 anos, com um acompanhamento médio de 3,8 anos. O ângulo pré-operatório médio da escoliose de $77^{\circ}$ foi inicialmente corrigido para $29^{\circ}$ e, no final do seguimento deteriorou para $34^{\circ}$. A média da obliquidade pélvica foi de $16^{\circ}$, corrigida no pós-operatório imediato para $10^{\circ} \mathrm{e}$, no pós-operatório tardio para $13^{\circ}$. A descompensação média do tronco foi de $117 \mathrm{~mm}$, inicialmente corrigida para $67 \mathrm{~mm}$ e, no final do seguimento, deteriorou para $98 \mathrm{~mm}$. Conclusões: Os pacientes no qual a instrumentação estendeu-se até a pelve, obtiveram melhores resultados em todas as mudanças relativas ao longo do tempo, quais sejam, escoliose, obliquidade pélvica e descompensação do tronco, com exceção da perda de correção da escoliose, que foi menor nos pacientes que não tiveram fixação da pelve.
\end{abstract}

Descritores: Mielomeningocele; Escoliose/etiologia; Escoliose/cirurgia.

\begin{abstract}
Objective: To evaluate the clinical and radiographic results of surgical treatment for paralytic scoliosis in myelomeningocele. Methods: A retrospective study by reviewing medical records and radiographs of patients with myelomeningocele, surgically treated between the years 1999 and 2009. Results: We analyzed the medical records and radiographs of 29 patients. The mean age at surgery was 12.2 years, with a mean follow-up of 3.8 years. The mean preoperative angle of scoliosis was $77^{\circ}$, initially adjusted to $29^{\circ}$ that, at the end of follow-up, deteriorated to $34^{\circ}$. The average of the pelvic obliquity was $16^{\circ}$, corrected in the immediate postoperative period to $10^{\circ}$, and in the late postoperative period to 13. The trunk decompensation average was $117 \mathrm{~mm}$, initially adjusted to $67 \mathrm{~mm}$ and, at final follow-up, deteriorated to $98 \mathrm{~mm}$. Conclusions: Patients in which the instrumentation was extended to the pelvis, obtained better results for all related changes over time, namely, scoliosis, pelvic obliquity and decompensation of the trunk, except for the loss of scoliosis correction that was lower in patients not undergoing pelvic fixation.
\end{abstract}

Keywords: Myelomeningocele, Scoliosis/etiology; Scoliosis/surgery.

\section{RESUMEN}

Objetivo: Evaluar los resultados clínicos y radiológicos del tratamiento quirúrgico de la escoliosis paralítica en la mielomeningocele. Métodos: Estudio retrospectivo mediante la revisión de historia clínica y estudios radiológicos de pacientes con mielomeningocele tratados quirúrgicamente entre 1999 y 2009. Resultados: Se revisaron las historias clínicas y las radiografías de 29 pacientes. El promedio de edad en el momento de la cirugía fue de 12,2 años, con un seguimiento promedio de 3,8 años. El ángulo preoperatorio medio de la escoliosis de $77^{\circ}$, fue corregido inicialmente a $29^{\circ}$, y al final del seguimiento empeoró a $34^{\circ}$. La media de la oblicuidad pélvica fue de $16^{\circ}$, corregido en el postoperatorio inmediato para $10^{\circ}$, y en el periodo postoperatorio tardío para $13^{\circ}$. El promedio de la descompensación del tronco fue de $117 \mathrm{~mm}$, inicialmente ajustado en $67 \mathrm{~mm}$ y en el seguimiento final se deterioró hasta $98 \mathrm{~mm}$. Conclusiones: Los pacientes en los que se extendió la instrumentación hasta la pelvis, obtuvieron mejores resultados para todos los cambios relacionados con el tiempo, o sea, escoliosis, oblicuidad pélvica y descompensación del tronco, excepto por la pérdida de corrección de la escoliosis, que fue menor en pacientes que no tenían la fijación de la pelvis.

Descriptores: Mielomeningocele; Escoliosis/etiologia; Esoliosis/cirugía.

\section{INTRODUÇÃO}

Devido ao aprimoramento do tratamento multidisciplinar realizado para as crianças portadoras de mielodisplasia nas últimas décadas, a expectativa e a qualidade de vida aumentaram, com muitas atingindo a idade adulta com bom nível funcional. A escolio- se é um problema freqüente nos pacientes com mielomeningocele (MMC), que pode ser de etiologia paralítica, congênita ou ambas. Quanto mais alto o nível motor e o disrafismo, mais grave o grau do desvio e a sua progressão ${ }^{1}$

Das deformidades ortopédicas que ocorrem nessas crianças,

1. Estagiário de Coluna do Hospital Evangélico de Curitiba, Hospital Infantil Pequeno Príncipe e Hospital São Vicente de Curitiba - Curitiba, PR, Brasil.

2. Chefe do Serviço de Coluna e Cirurgião de Coluna do Serviço de Ortopedia eTraumatologia do Hospital Infantil Pequeno Príncipe - Curitiba, PR, Brasil.

3. Cirurgião de Coluna do Serviço de Ortopedia eTraumatologia do Hospital Infantil Pequeno Príncipe de Curitiba (HIPP) - Curitiba, PR, Brasil.

4. Chefe do Serviço de Coluna e Cirurgião de Coluna do Hospital Evangélico de Curitiba e Hospital São Vicente de Curitiba - Curitiba, PR, Brasil.

5. Cirurgião de Coluna do Hospital São Vicente de Curitiba - Curitiba, PR, Brasil. 
a escoliose é uma das mais difíceis de tratar. Como é comum a alteração sensitiva e motora, não é raro que apresentem áreas de pressão nas regiões de apoio e escaras. Quando ocorre a progressão da curva com a presença de obliquidade pélvica, pode haver dificuldade para sentar com a necessidade de apoiar os membros superiores para manter-se sentada, o que dificulta freqüentar a escola e utilização das mãos. Como parte da reabilitação, torna-se necessária a correção e estabilização da deformidade. Uma coluna vertebral estável e equilibrada permite o melhor aproveitamento da cirurgia de membros inferiores e liberação das mãos da necessidade de apoio ${ }^{2}$.

A escoliose paralítica deve ser avaliada e tratada de forma distinta da congênita. A magnitude e a progressão da curva paralítica estão diretamente relacionadas ao nível da paralisia e à idade do paciente no momento do aparecimento ${ }^{3}$. A história natural da escoliose associado com MMC foi relatada por diversos autores, tendo uma prevalência aproximada de $88 \%$ de escoliose no nível torácico, 81\% na área lombar alta, 23\% na área lombar baixa, e 9\% na área sacral ${ }^{2,4}$

Crianças portadoras de MMC, que têm paraplegia ou fraqueza da musculatura da coluna vertebral decorrente de alterações medulares, desenvolvem uma escoliose precoce e mais grave com característica paralítica e obliquidade pélvica ${ }^{1,4}$. Ao nascimento, no plano coronal, a coluna geralmente está alinhada, mas com o desenvolvimento, a postura sentada e o ortostatismo, ocorre o colapso devido à ação da gravidade, que continua a deteriorar-se até que o gradil costal pressione a crista ilíaca. A deformidade é uma típica lordo-escoliose longa toracolombar que se estende da região torácica superior até a pelve, com obliquidade pélvica. Com a progressão de deformidade, a criança passa a sentar em uma nádega, precisando de seus braços para apoio, desenvolvendo áreas de pressão e escaras, devido à alteração de sensibilidade ao nível do quadril e ísquio ${ }^{5}$. Escoliose progressiva com descompensação do tronco associada e obliquidade pélvica colocam em risco as atividades da vida diária desses pacientes, podendo evoluir com comprometimento cardíaco, respiratório e de órgãos intra-abdominais ${ }^{3}$

Tratamento conservador com órteses é ineficaz como tratamento isolado, porém pode ser ocasionalmente e temporariamente utilizada como um método para melhorar o equilíbrio sentado no paciente de baixa idade, postergando a correção cirúrgica para uma idade mais apropriada. O único tratamento para as curvas progressivas é a correção e estabilização cirúrgica da coluna vertebral. O objetivo da cirurgia da escoliose associada a MMC é evitar deformidade espinhal progressiva, melhorar o equilíbrio sentado, e prevenir ou curar úlceras de pressão na pele. Os ganhos secundários da cirurgia incluem a melhoria da mobilidade, da capacidade de transferência e das atividades da vida diária ${ }^{5}$.

O tratamento cirúrgico destas deformidades é um grande desafio. É tecnicamente exigente e, demanda uma técnica apurada, conhecimento da anatomia alterada, para reduzir a morbidade e intercorrências decorrentes do ato cirúrgico. Liberação cirúrgica e artrodese anterior e posterior combinadas, seguida por instrumentação posterior estendendo-se até sacro, tornaram-se o procedimento de escolha para o tratamento dessas deformidades complexas com obliqüidade pélvica. Obtendo maior correção da curva e reduzindo a incidência de pseudoartrose no segmento do disrafismo $0^{3,5-9}$.

A necessidade de instrumentação anterior é controversa. Atualmente, com os sistemas de fixação segmentar com parafuso pedicular, osteotomias e ressecções vertebrais, a necessidade de uma abordagem anterior também vem sendo questionada². A inclusão do sacro na artrodese resulta em melhor correção de obliqüidade pélvica, que é estável e confiável, melhorando o equilíbrio sentado. Contudo, a perda de mobilidade lombo-sacra, proveniente da fusão sacral, pode limitar ou dificultar consideravelmente as transferências e outras atividades da reabilitação ${ }^{6}$

\section{OBJETIVOS}

\section{Geral}

Avaliar as variáveis clínicas e os resultados radiográficos dos pacientes portadores de Escoliose Paralítica por Mielomeningocele (MMC), tratados cirurgicamente entre os anos de 1999 e 2009, no Hospital Infantil Pequeno Príncipe, em Curitiba - PR, por um único cirurgião.

\section{Específicos}

Avaliar as diferentes técnicas cirúrgicas utilizadas, além das complicações inerentes a cada uma;

Avaliar as variáveis clínicas (idade, sexo, nível de paralisia, tempo de seguimento, etc.) e os resultados radiográficos (ângulo de escoliose, descompensação do tronco e obliqüidade pélvica) dos pacientes no pré-operatório, pós-operatório imediato e pós-operatório tardio;

Determinar a correção primária, a correção de médio prazo e a perda de correção, obtidas nos pacientes;

Avaliar e identificar os fatores que possam interferir no resultado cirúrgico final.

\section{AMOSTRA}

Trinta e seis pacientes, com diagnóstico de escoliose paralítica por mielomeningocele, foram submetidos a tratamento cirúrgico entre os anos de 1999 e 2009, no Hospital Infantil Pequeno Príncipe, em Curitiba - PR. Destes, apenas 28 (78\%) preencheram os critérios de inclusão e, seus dados foram analisados neste estudo.

\section{Critérios de Inclusão}

Ter diagnóstico de escoliose paralítica por mielomeningocele;

Ter sido submetido ao tratamento cirúrgico entre os anos de 1999 e 2009;

Ter realizado o seguimento pós-operatório no Hospital Infantil Pequeno Príncipe;

Constar em seu prontuário as informações necessárias para a completa coleta dos dados (descrição cirúrgica, radiografias pré e pós-operatórias, etc.)

\section{Critérios de Exclusão}

Ter diagnóstico de escoliose de qualquer outro tipo ou causa que não a paralítica por mielomeningocele;

Ter sido submetido ao tratamento cirúrgico antes de 1999 ou depois de 2009;

Não ter realizado o seguimento pós-operatório no Hospital Infantil Pequeno Príncipe;

Não constar em seu prontuário as informações necessárias para a completa coleta dos dados (descrição cirúrgica, radiografias pré e pós-operatórias, etc.).

\section{MATERIAL E MÉTODO}

Foi realizada uma revisão retrospectiva de prontuários e radiografias dos pacientes com diagnóstico de escoliose paralítica por MMC, submetidos a tratamento cirúrgico, realizado por apenas um cirurgião de coluna, no Hospital Infantil Pequeno Príncipe, durante o período de janeiro de 1999 a dezembro de 2009. O tamanho da amostra foi de 29 pacientes.

Os seguintes dados clínicos foram obtidos a partir de prontuários: 1) idade no momento da cirurgia; 2) gênero; 3) nível de paralisia, (segmento mais caudal totalmente inervado) e última vértebra intacta 4) técnica cirúrgica utilizada; 5) extensão da artrodese; 6) complicações e 7) tempo de seguimento.

O ângulo da escoliose (método de Cobb, em graus), descompensação do tronco (em milímetros) e obliquidade pélvica (em graus) foram medidos através de radiografias que foram feitas no pré-operatório, pós-operatório imediato e pós-operatório tardio.

Dos ângulos da escoliose, foram determinados: 1) Correção 
Primária - definida como o valor pré-operatório menos o valor pós-operatório imediato; 2) Correção Tardia - definida como o valor pré-operatório menos o valor pós-operatório tardio; e 3) Perda de Correção - definida como o valor pós-operatório tardio menos o valor pós-operatório imediato. As mudanças relativas (em porcentagem) foram calculadas de acordo com as seguintes fórmulas:

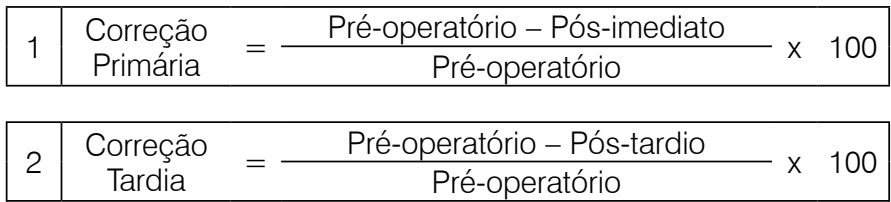

\begin{tabular}{|c|c}
\hline 3 & $\begin{array}{l}\text { Perda de } \\
\text { Correção }\end{array}=\frac{\text { Pós-tardio }- \text { Pós-imediato }}{\text { Pré-operatório - Pós-imediato }} \times 100$ \\
\hline
\end{tabular}

\section{RESULTADOS}

Trinta e seis pacientes, com diagnóstico de escoliose paralítica por mielomeningocele, foram submetidos a tratamento cirúrgico entre os anos de 1999 e 2009, no Hospital Infantil Pequeno Príncipe, em Curitiba, PR. Destes, 29 (80\%) pacientes preencheram os critérios de inclusão e, seus dados foram analisados neste estudo.

Os dados clínicos dos pacientes estão representados na Tabela 1. Doze pacientes (41\%) eram do gênero masculino e 17 (59\%) do feminino. A idade no momento da cirurgia foi em média 12,2 anos de idade (variando de 8,8 anos a 17,3 anos) e, tiveram um acompanhamento em média de 3,8 anos (de 2 anos a 11,3 anos). O nível da paralisia encontrado nos pacientes foi: 1) 12 com nível torácico (41\%); 2) $9 \mathrm{com}$ nível lombar alto (31\%); 3) 7 com nível lombar baixo (24\%) e; 4) apenas 1 com nível sacral (3\%).

As técnicas cirúrgicas realizadas podem ser descritas simplificadamente, como apenas por via posterior, utilizadas em apenas cinco pacientes (17\%) e, as por dupla via, anterior e posterior, utilizadas na maioria dos pacientes (24 - 83\%). Independentemente da técnica cirúrgica utilizada, o número de vértebras incluídas na artrodese foi, em média, de 14 (variando de 10 a 17 vértebras).

De todos os pacientes, sete (24\%) evoluíram com cinco tipos diferentes de complicações: dois pacientes (7\%) tiveram pseudo-artrose, mas apenas um deles necessitou de cirurgia de revisão; três (10\%) evoluíram com infecção da ferida cirúrgica da via posterior, sendo que um deles também evoluiu com psoíte e trombose venosa profunda da veia femoral, após a abordagem anterior e; um paciente, sem história prévia da alergia, apresentou reação anafilática ao látex, durante a abordagem anterior, sendo necessário prorrogar o procedimento pela via posterior para outro momento.

Os dados radiográficos dos pacientes estão representados na Tabela 2. No pré-operatório, a média do ângulo de Cobb para escoliose nos pacientes foi de $77^{\circ}$ (variando de $39^{\circ}$ a $115^{\circ}$ ); a média da obliquidade pélvica foi de $16^{\circ}$ (de $1^{\circ}$ a $49^{\circ}$ ) e; a descompensação

Tabela 1. Dados demográficos e clínicos dos pacientes.

\begin{tabular}{c|c|c|c}
\hline \multicolumn{2}{c|}{ Dados Clínicos } & Total ou Média & $\%$ ou Intervalo \\
\hline \multirow{2}{*}{ Gênero } & Masculino & 12 & $(41 \%)$ \\
\cline { 2 - 4 } & Feminino & 17 & $(59 \%)$ \\
\hline \multicolumn{2}{|c|}{ Idade na cirurgia } & 12,2 anos & $(8,8-17,3$ anos) \\
\hline \multicolumn{2}{c}{ Tempo de Seguimento } & 3,8 anos & $(2$ anos $-11,3$ anos $)$ \\
\hline \multirow{4}{*}{ Nível de paralisia } & Torácico & 12 & $(41 \%)$ \\
\cline { 2 - 4 } & Lombar Alto & 9 & $(31 \%)$ \\
\cline { 2 - 4 } & Lombar Baixo & 7 & $(24 \%)$ \\
\cline { 2 - 4 } & Sacral & 1 & $(3 \%)$ \\
\hline \multirow{2}{*}{ Técnica Cirúrgica } & VP & 5 & $(17 \%)$ \\
\cline { 2 - 4 } & VA + VP & 24 & $(83 \%)$ \\
\hline \multirow{2}{*}{ Número de Vértebras na Artrodese } & 14 & $(10-17)$ \\
\hline \multirow{2}{*}{ Ocorrência de Complicações } & Sim & 7 & $(24 \%)$ \\
\cline { 2 - 4 } & Não & 22 & $(76 \%)$ \\
\hline
\end{tabular}

do tronco em média foi de $117 \mathrm{~mm}$ (de 5 a $283 \mathrm{~mm}$ ). No pós-operatório imediato, os valores do ângulo de Cobb para escoliose teve a média de $29^{\circ}$ (entre $4^{\circ}$ e $64^{\circ}$ ); a média da obliquidade pélvica foi de $10^{\circ}$ (variando de $2^{\circ}$ a $2^{\circ}$ ) e; a descompensação do tronco em média foi de $67 \mathrm{~mm}$ (de 2 a $213 \mathrm{~mm}$ ). No pós-operatório tardio, as médias do ângulo de Cobb para escoliose foi de $34^{\circ}\left(5^{\circ}\right.$ a $\left.72^{\circ}\right)$; da obliqüidade pélvica foi de $13^{\circ}\left(0\right.$ a $\left.36^{\circ}\right)$ e; da descompensação do tronco foi de $98 \mathrm{~mm}$ (variando de 6 a $265 \mathrm{~mm}$ ).

Dos ângulos da escoliose, foram determinadas as mudanças relativas, onde todos os pacientes apresentaram correção primária, que em média foi de $60 \%$ (variando de $7 \%$ a 93\%) e; correção tardia em média foi de 54\% (variando de $2 \%$ a 94\%). Apenas 17 pacientes (58\%) apresentaram perda de correção com uma média de 15\% (variando de $5 \%$ a $67 \%$ ) e de $11 \%$ (variando de $-21 \%$ a $67 \%$ ) entre todos os 28 pacientes. Para a obliquidade pélvica os valores foram: $7 \%$ de correção primária; -19\% de Correção Tardia e; 31\% de perda da correção. Para a descompensação do tronco os valores da correção primária, correção tardia e perda da correção foram de $24 \%,-21 \%$ e $56 \%$, respectivamente (Tabela 3 ).

Quando separamos os pacientes naqueles que realizaram instrumentação incluindo a pelve (Grupo I - N=7) e aqueles na qual a instrumentação não incluiu a pelve (Grupo $\|-N=22$ ), observamos melhores resultados nos pacientes do Grupo I, para todas as mudanças relativas ao longo do tempo, escoliose, obliquidade pélvica e descompensação do tronco, com exceção da perda de correção da escoliose que foi menor nos pacientes do Grupo II (Tabela 4).

Tabela 2. Dados radiográficos dos pacientes.

\begin{tabular}{c|c|c|c}
\hline \multirow{4}{*}{ Pré-operatório } & Escoliose (ângulo de Cobb) & Média & Intervalo \\
\cline { 2 - 4 } & Obliquidade Pélvica (graus) & $17^{\circ}$ & $\left(39^{\circ}-115^{\circ}\right)$ \\
\cline { 2 - 4 } & Descompensação do Tronco $(\mathrm{mm})$ & 117 & $\left(1^{\circ}-49^{\circ}\right)$ \\
\hline \multirow{4}{*}{ Pós-operatório imediato } & Escoliose (ângulo de Cobb) & $29^{\circ}$ & $\left(4^{\circ}-64^{\circ}\right)$ \\
\cline { 2 - 4 } & Obliquidade Pélvica (graus) & $10^{\circ}$ & $\left(2^{\circ}-25^{\circ}\right)$ \\
\cline { 2 - 4 } & Descompensação do Tronco (mm) & 67 & $(2-213)$ \\
\hline \multirow{3}{*}{ Pós-operatório tardio } & Escoliose (ângulo de Cobb) & $34^{\circ}$ & $\left(5^{\circ}-72^{\circ}\right)$ \\
\cline { 2 - 4 } & Obliquidade Pélvica (graus) & $13^{\circ}$ & $\left(0-36^{\circ}\right)$ \\
\cline { 2 - 4 } & Descompensação do Tronco (mm) & 98 & $(6-265)$ \\
\hline
\end{tabular}

Tabela 3. Mudanças relativas de escoliose, obliquidade pélvica e descompensação do tronco dos 28 pacientes.

\begin{tabular}{c|c|c|c}
\hline & $\begin{array}{c}\text { Correção Primária } \\
(\mathbf{\%})\end{array}$ & $\begin{array}{c}\text { Correção Tardia } \\
(\mathbf{\%})\end{array}$ & $\begin{array}{c}\text { Perda de Correção } \\
(\mathbf{\%})\end{array}$ \\
\hline Escoliose & $60(7-93)$ & $54(2-94)$ & $11(-21-67)$ \\
\hline Obliquidade Pélvica & $7(-400-90)$ & $-19(-400-100)$ & $31(-200-500)$ \\
\hline Descompensação do Tronco & $24(-380-99)$ & $-21(-460-97)$ & $56(-660-1240)$ \\
\hline
\end{tabular}

Tabela 4. Mudanças relativas de escoliose, obliquidade pélvica e descompensação do tronco para inclusão (Grupo I) e não inclusão (Grupo II) da pelve na instrumentação.

\begin{tabular}{c|c|c|c|c|c|c}
\hline \multirow{2}{*}{} & \multicolumn{2}{|c|}{$\begin{array}{c}\text { Correção Primária } \\
(\%)\end{array}$} & \multicolumn{2}{c|}{$\begin{array}{c}\text { Correção Tardia } \\
(\%)\end{array}$} & $\begin{array}{c}\text { Perda de Correção } \\
(\%)\end{array}$ \\
\cline { 2 - 7 } & Grupo I & Grupo II & Grupo I & Grupo II & Grupo I & Grupo II \\
\hline Escoliose & 64 & 59 & 58 & 54 & 17 & 9 \\
\hline Obliquidade Pélvica & 52 & -7 & 58 & -43 & -15 & 45 \\
\hline Descompensação do Tronco & 68 & 9 & 56 & -45 & 21 & 68 \\
\hline
\end{tabular}

\section{DISCUSSÃO}

Escoliose grave e descompensada, em pacientes com MMC continua a ser um desafio para os cirurgiões de coluna. $\mathrm{O}$ tratamento cirúrgico visa melhorar o alinhamento coronal (ângulo de escoliose) e sagital (cifose e lordose), que pode apresentar uma perda da correção no seguimento tardio. O objetivo deste estudo foi analisar um grupo de pacientes, com escoliose paralítica associada à MMC, quanto às suas variáveis clínicas e seus resultados 
radiográficos, submetidos ao tratamento cirúrgico.

No passado, muitas técnicas cirúrgicas diferentes foram usadas para o tratamento cirúrgico da escoliose paralítica associada a MMC. Fusão anterior e posterior é amplamente recomendada ${ }^{2,3,5-9}$. Osebold et al. ${ }^{8}$ relataram 17 pacientes com uma escoliose média pré-operatória de $81^{\circ}$, com ganho médio de $62 \%$. McMaster ${ }^{5}$ e Parsch et al. ${ }^{3}$ relataram valores semelhantes $\left(98^{\circ}\right.$ para $70 \%$ e $92^{\circ}$ para 59\%, respectivamente). Estes resultados são bem comparáveis com os achados deste estudo ( $77^{\circ}$ melhorou em média $\left.60 \%\right)$.

Para obtenção da maior correção possível nas escolioses graves, muitos autores recomendam uma liberação extensa, tanto anterior como posterior ${ }^{3,5,8}$. Parsch et al. ${ }^{3}$ mostrou que tanto a correção primária como a correção tardia da escoliose melhorou significativamente com a extensão da artrodese. Estas recomendações estão de acordo com os achados desse estudo, que teve em média 14 vértebras incluídas na área de artrodese.

Inclusão da pelve na artrodese resulta em melhor correção de obliquidade pélvica, é uma fixação mais estável e confiável e, assim, melhora o equilíbrio sentado. McMaster ${ }^{5}$, Osebold et al. ${ }^{8}$ e Parsch et al. ${ }^{3}$ recomendam que em pacientes com escoliose grave, e marcada descompensação do tronco ou obliquidade pélvica, a artrodese deve ser estendida e incluir a junção lombo-sacral para estabelecer um tronco compensado sobre uma pelve nivelada. Já Wild et al. ${ }^{6}$ observaram a correção da obliqüidade pélvica espontaneamente quando a deformidade escoliótica foi adequadamente tratada com a artrodese e instrumentação, sem inclusão de sacro, oferecendo a estes pacientes menor comprometimento da mobilidade. Neste estudo, sete pacientes (24\%) tiveram a instrumentação até a pelve, e foram os que obtiveram melhores resultados na correção da escoliose, obliqüidade pélvica e descompensação do tronco, em todas as mudanças relativas ao longo do seguimento, com exceção da perda de correção da escoliose, que foi maior nesses pacientes.

A taxa de complicações encontrada neste estudo (24\%), relacio- nadas ao paciente ou ao implante/técnica cirúrgica, está de acordo com a relatada na literatura $(18 \%-57 \%)^{2,3,5,8,9}$. A pseudo-artrose é uma das principais complicações que pode ocorrer e, que leva a perda da correção, falha do implante e, consequentemente, re-operações. A incidência de pseudo-artrose na literatura pode ter valores tão altos quanto $50 \%$, dependendo da técnica cirúrgica utilizada ${ }^{2,3,5,6,8,9}$. Nesse estudo, a pseudo-artrose teve uma incidência total de $7 \%$, independentemente da técnica cirúrgica utilizada, sendo que apenas um paciente necessitou de cirurgia de revisão.

\section{CONCLUSÃO}

O tratamento cirúrgico da escoliose paralítica associada à mielomeningocele é uma tarefa árdua e complexa, com necessidade de um cirurgião experiente, mas com grande benefício para essas crianças com graves deficiências e suas famílias. Há, no entanto, um grande potencial para complicações e um grande cuidado, bem como uma vasta experiência são necessários.

O tratamento desses pacientes deve incluir fechamento precoce do saco dural, derivação para controlar a hidrocefalia, tratamento das anomalias do trato urinário, e cuidados com a grande variedade de graves complicações cirúrgicas que possam ocorrer. Uma equipe multidisciplinar é, portanto, necessária para o manejo destes pacientes. Apesar das técnicas e resultados terem melhorado muito nos últimos anos, outros problemas permanecem, entre eles encontrar a melhor indicação para a abordagem anterior, para a utilização das ressecções vertebrais e o risco/benefício para inclusão da pelve na instrumentação.

Os dados encontrados nesse estudo são semelhantes à literatura, porém o pequeno número de pacientes que realizaram instrumentação que incluía a pelve, pode ter levado a piores resultados no total da perda da correção e, principalmente naqueles pacientes que a pelve não foi incluída na instrumentação.

\section{REFERÊNCIAS}

1. Piggott $\mathrm{H}$. The natural history of scoliosis in myelodysplasia. J Bone Joint Surg Br. 1980:62(1):54-8

2. Banit DM, Iwinski HJ Jr, Talwalkar V, Johnson M. Posterior spinal fusion in paralytic scoliosis and myelomeningocele. J Pediatr Orthop. 2001;21(1):117-25.

3. Parsch D, Geiger F, Brocai DR, Lang RD, Carstens C. Surgical management of paralytic scoliosis in myelomeningocele. J Pediatr Orthop B. 2001;10(1):10-7.

4. Shurtleff DB, Goiney R, Gordon LH, Livermore N. Myelodysplasia: the natural history of kyphosis and scoliosis. A preliminary report. Dev Med Child Neurol Suppl. 1976;(37):126-33.

5. McMaster MJ. Anterior and posterior instrumentation and fusion of thoracolumbar scoliosis due to myelomeningocele. J Bone Joint Surg Br. 1987;69(1):20-5.
6. Wild $A$, Haak $H$, Kumar $M$, Krauspe R. Is sacral instrumentation mandatory to address pelvic obliquity in neuromuscular thoracolumbar scoliosis due to myelomeningocele? Spine (Phila Pa 1976). 2001;26(14):E325-9.

7. Osebold WR. Stability of myelomeningocele spines treated with the mayfield two-stage anterior and posterior fusion technique. Spine (Phila Pa 1976). 2000;25(11):1344-51.

8. Osebold WR, Mayfield JK, Winter RB, Moe JH. Surgical treatment of paralytic scoliosis associated with myelomeningocele. J Bone Joint Surg Am. 1982;64(6):841-56.

9. Sponseller PD, Young AT, Sarwark JF, Lim R. Anterior only fusion for scoliosis in patients with myelomeningocele. Clin Orthop Relat Res. 1999;(364):117-24 